\title{
Pulmonary Neuroendocrine Tumor with Pancreatic Metastasis: A Case Report
}

\author{
Weihua Wang $\mathrm{BS}^{1}$ and Yuling Tong $\mathrm{MS}^{2 *}$ \\ ${ }^{1}$ Department of Internal Medicine, The People'S Hospital of Suichang County, Lishui, China \\ ${ }^{2}$ Department of General Practice/Health Management Center, The $2^{\text {nd }}$ Affiliated Hospital of Zhejiang University, School of \\ Medicine, Hangzhou, China \\ *Corresponding author: Yuling Tong MS, Department of General Practice/Health Management Center, The $2^{\text {nd }}$ affiliated hospital of \\ Zhejiang university, school of medicine, China
}

ARTICLE INFO

Received: 㗀 July 22, 2021

Published: 幽 August 02, 2021

Citation: Weihua Wang BS, Yuling Tong MS. Pulmonary Neuroendocrine Tumor with Pancreatic Metastasis: A Case Report. Biomed J Sci \& Tech Res 37(4)-2021. BJSTR. MS.ID.006036.

Keywords: Pulmonary Neuroendocrine Tumor; Pancreatic Metastasis; Multidisciplinary Discussion; Chemotherapy; Diagnosis and Treatment; Case Report
ABSTRACT

pancreatic metastases with abdominal pain were rarely seen as the first symptom in patients of pulmonary neuroendocrine tumor. We herein reported a case of a 56-year-old man who presented epigastric pain and slightly elevated CA19-9 levels. Histopathology confirmed diagnosis of extensive-stage small cell lung cancer with multiple metastases of pancreas, intracranial and left chest wall. The patient underwent six courses of chemotherapy (Etoposide and Cisplatin) and one course of cranial radiotherapy and had a total survival of 9 months. The possibility of rare tumors should be considered in patients with multiple systemic metastases but were in good general condition.

Abbreviations: CEA: Carcinoembryonic Antigen; CA19-9: Carbohydrate Antigen 19-9; MDT: Multidisciplinary Discussion; SCLC: Small Cell Lung Cancer; LCNEC: Large Cell Neuroendocrine Cancer; TC: Typical Carcinoid; AC: Atypical Carcinoid; TTF1: Thyroid Transcription Factor 1; VALG: Veterans Administration Cancer Study Group; SD: Stable Disease

\section{Introduction}

Neuroendocrine tumors are a group of tumors that share morphological, immunohistochemical and ultrastructural pathology and molecular features. The incidence of pulmonary neuroendocrine tumor is gradually increasing, leading to distant metastasis of liver, bone, brain, and adrenal gland [1]. However, pancreatic metastasis is rare, which accounted for about $0.7 \%$ [2]. In addition, majority of patients with metastatic pancreatic cancer had normal serum carbohydrate antigen 19-9 (CA19-9) levels [3]. We herein reported a case report of pancreatic metastases from pulmonary neuroendocrine tumor with abdominal symptoms and elevated serum CA19-9 levels. Previous studies were reviewed in order to provide some experiences regarding the diagnosis and treatment of such diseases.

\section{Case Presentation}

A 56-year-old male was admitted to the People's Hospital of Suichang County on May 10, 2019 with epigastric dull pain radiating into the posterior back for more than 20 days and had no obvious aggravation and relief factors. There were no nausea or any change in bowel movement habits and weight, and no pulmonary and nerve system symptoms were observed. Some medications for gastritis were taken by him, but no effect was achieved. The patient denied previous medical history of cardiovascular disease, pulmonary disease, gastrointestinal disease, tuberculosis, and denied history of food and drug allergy. He has smoking history of 40 cigarettes/day for more than 30 years (smoking index > 1200). A $4 \mathrm{~cm} * 4 \mathrm{~cm}$ mass was found in the left chest wall, with clear 
boundary, hard texture, and well activity. His abdomen was soft, with slight tenderness in the right upper abdomen. No enlarged systemic superficial lymph nodes were observed. No positive signs of cardiovascular system, pulmonary system and nervous system were found. Laboratory tests revealed elevated levels of carcinoembryonic antigen (CEA) $9.82 \mathrm{ng} / \mathrm{ml}$ (reference range: $0-5 \mathrm{ng} / \mathrm{ml}$ ), and CA19-9 44.50U/ml(reference range: $0-37 \mathrm{U} / \mathrm{ml}$ ).
Enhanced CT scan showed multiple pancreatic tumors with lymph node metastasis in the retroperitoneum. Pulmonary CT showed left lung tumor with multiple metastasis (of lung in the left chest wall and lymph nodes in the hilum, mediastinum, axilla). (Figure 1) Cranial MR suggested multiple abnormal signal nodules in both cerebellar hemispheres and considered it as metastatic.

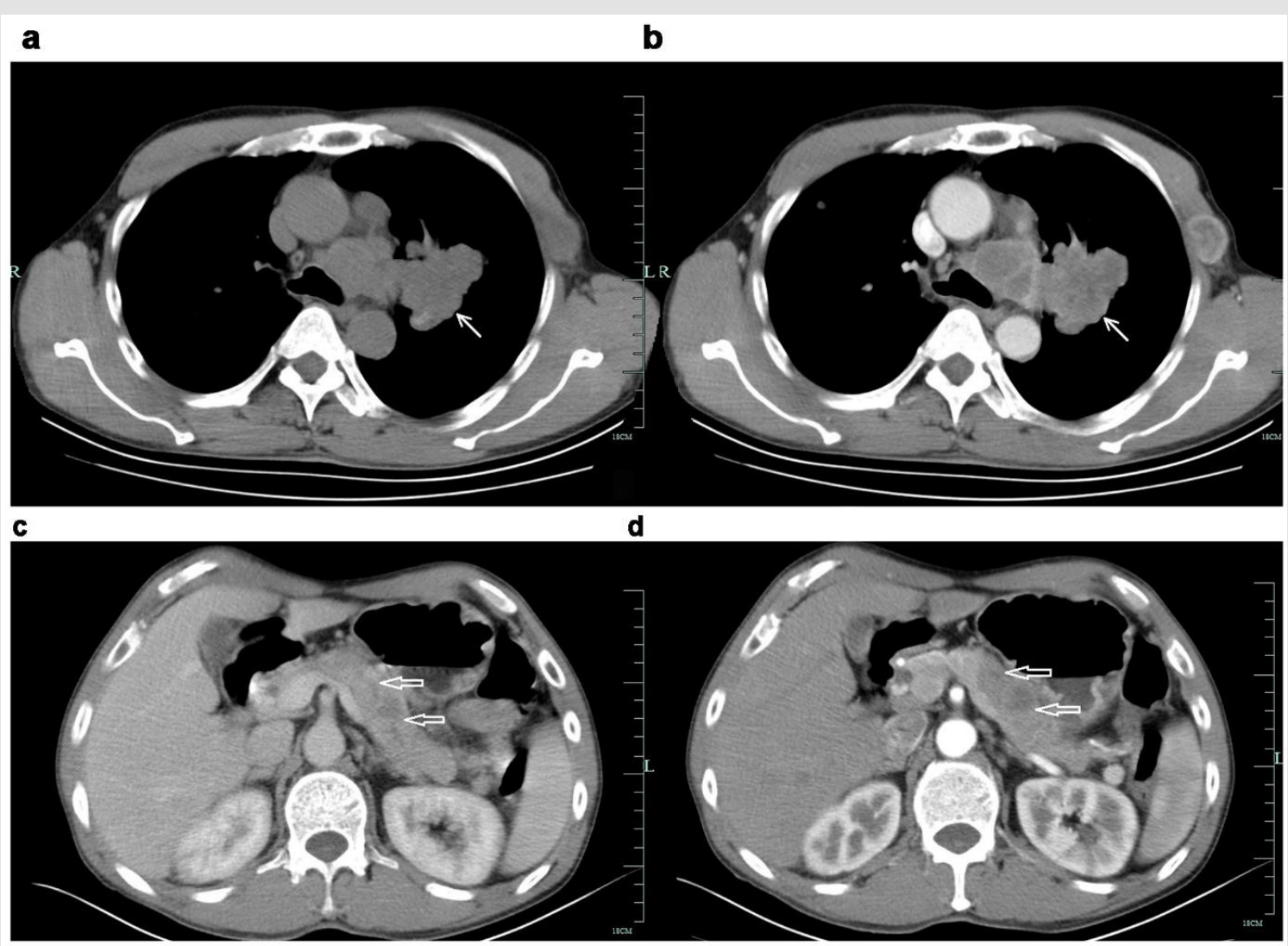

Figure 1: Enhancement CT of the patient.

a. a\&b: Irregularly lobulated uniform-density soft tissue mass was found in the lateral hilar region of the upper lobe of the left lung, with mild enhancement in enhanced scanning. Bronchial stenosis and occlusion of the upper lobe of the left lung ( $)$.

b. c\&d: Multiple low-density lesions in the body and tail of enlarged pancreas, with delayed enhancement in enhanced scanning. The distal pancreatic duct was slightly dilated. The local fat space was not clear, and no enlarged lymph node was observed $(\Leftarrow)$.

A multidisciplinary discussion (MDT) was conducted and concluded that the patient had a short course of disease and was generally in good condition, while radiology results suggested multiple occupying lesions in lung and pancreas, and advanced malignant tumors with multiple metastases. Metastatic process and elevated CA19-9 levels revealed lung metastasis from pancreatic cancer. However, according to radiological images, an irregular lobulated uniform-density soft tissue mass in the left lung was observed and was tended to be primary. Pathological diagnosis is considered extremely important. It is difficult to obtain a biopsy of lung and pancreatic lesion, for the limitation of medical equipment (such as endoscopic ultrasonography) in county hospital, and the patient was reluctant to be transferred. So coarse needle puncture biopsy of left chest wall mass was performed. The pathological results suggested (left chest wall puncture biopsy) neuroendocrine tumor (poorly differentiated neuroendocrine carcinoma), confirming it to be the origin of small cell carcinoma of the lung. Immunohistochemical results showed CgA (+), Syn (+), CD56 (+), CK18 ( \pm$),$ CK $( \pm)$, CA199 (+), P53 (-), P63(-), P40 (-), Ki-67 (50\%), ERCC-1 (+), CK7 (-), TTF1 (+), and Napsin-A (-). (Figures 2 \& 3) Thus, the final diagnosis revealed extensive-stage small cell lung cancer with multiple metastases of pancreas, intracranial and left chest wall. The patient currently underwent six courses of chemotherapy and one course of cranial radiotherapy (30Gy/10F). 
The chemotherapy regimen used was etoposide $\left(100 \mathrm{mg} / \mathrm{m}^{2}, \mathrm{~d} 1-3\right)$ combined with cisplatin $\left(75 \mathrm{mg} / \mathrm{m}^{2}, \mathrm{~d} 1\right)$, and each course lasted for 21 days, according to the NCCN guidelines. After the first-course chemotherapy, the patient's symptoms were improved. Follow-up CT after four courses of chemotherapy showed that the lesions in the lung, pancreas and left chest wall were obviously shrunken. (Figure4) The patient was closely followed up. The patient's lung lesions developed later and eventually died of respiratory failure. The total survival time is 9 months. Patient's informed consent was obtained to publish this case report.

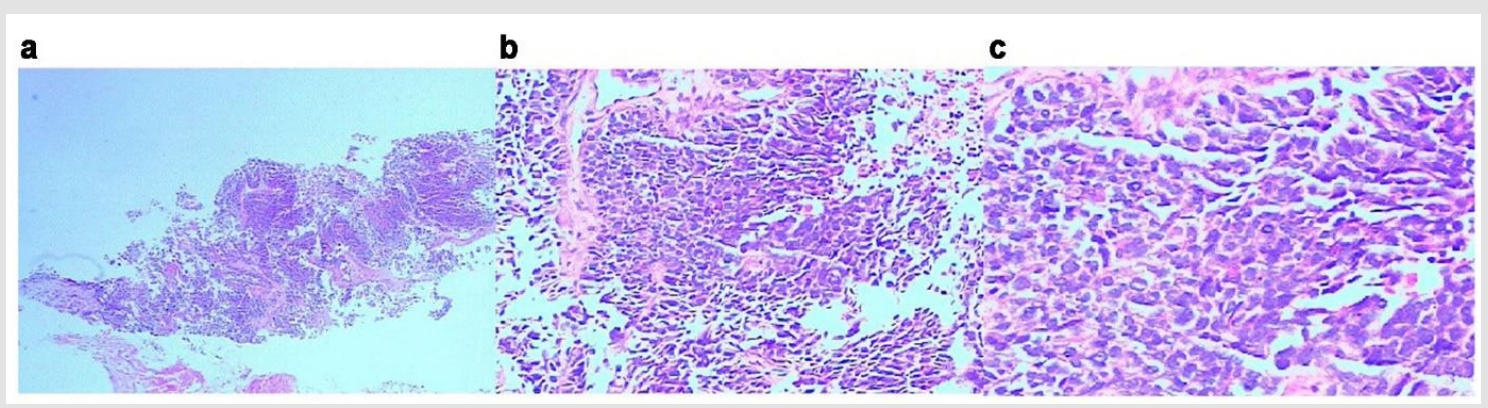

Figure 2: Pathological biopsy of the left chest wall.

a. HE, $x 2$.

b. HE, $\times 200$.

c. HE, $x 400$.

The cell layers were clustered and nested, and organ-like structures were seen. The cells were small with less cytoplasm, unclear nucleoli, partial vacuoles in the nucleus and chromatin. Karyokinesis was observed with local coagulation necrosis.

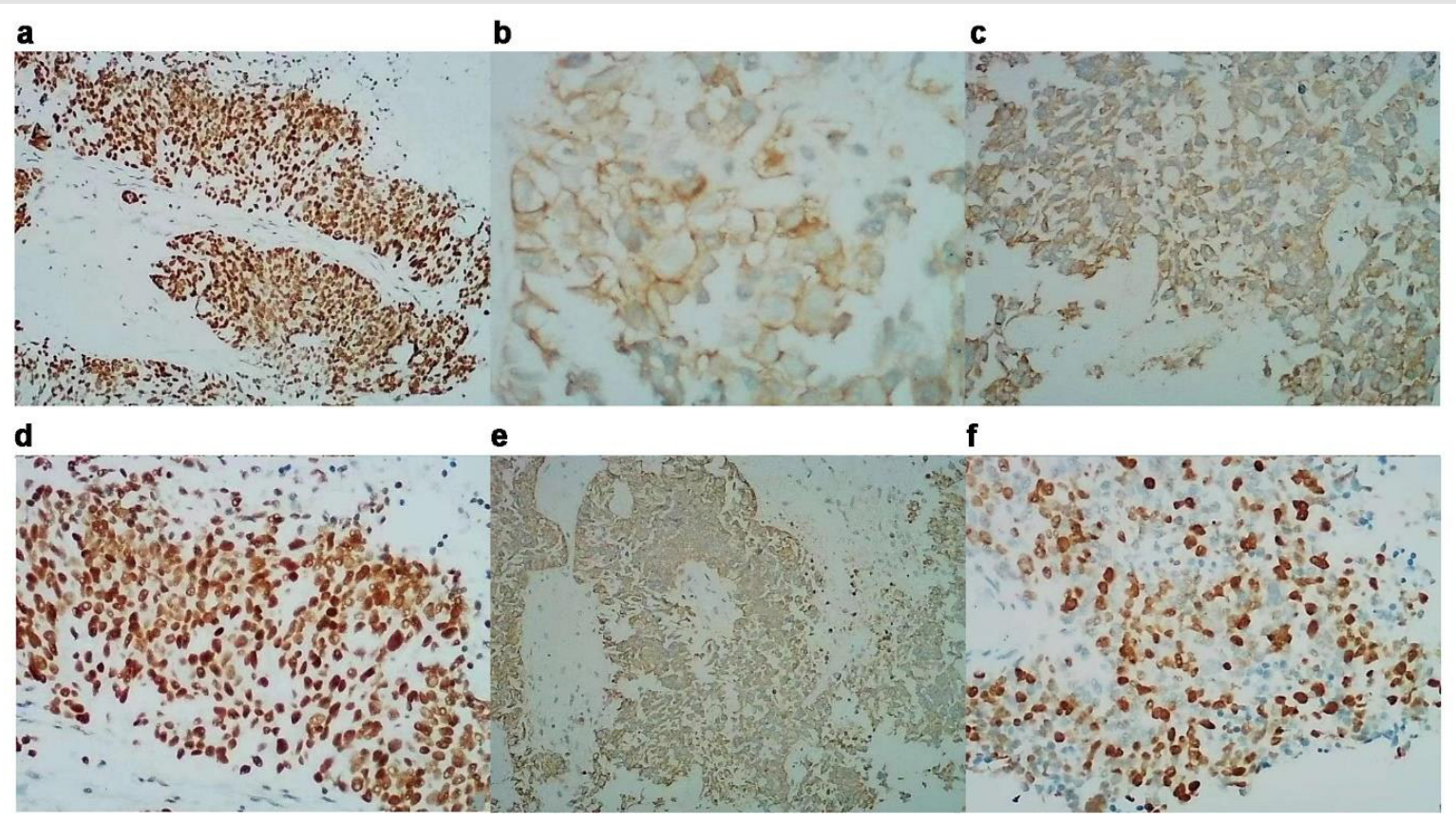

Figure 3: Immunohistochemistry of the patient
a. $\mathrm{CgA}(+)$;
b. $\operatorname{Syn}(+)$;
c. CD56(+);
d. TTF-1(+);
e. $\mathrm{CK}( \pm)$;
f. $\operatorname{Ki} 67(50 \%)$ 


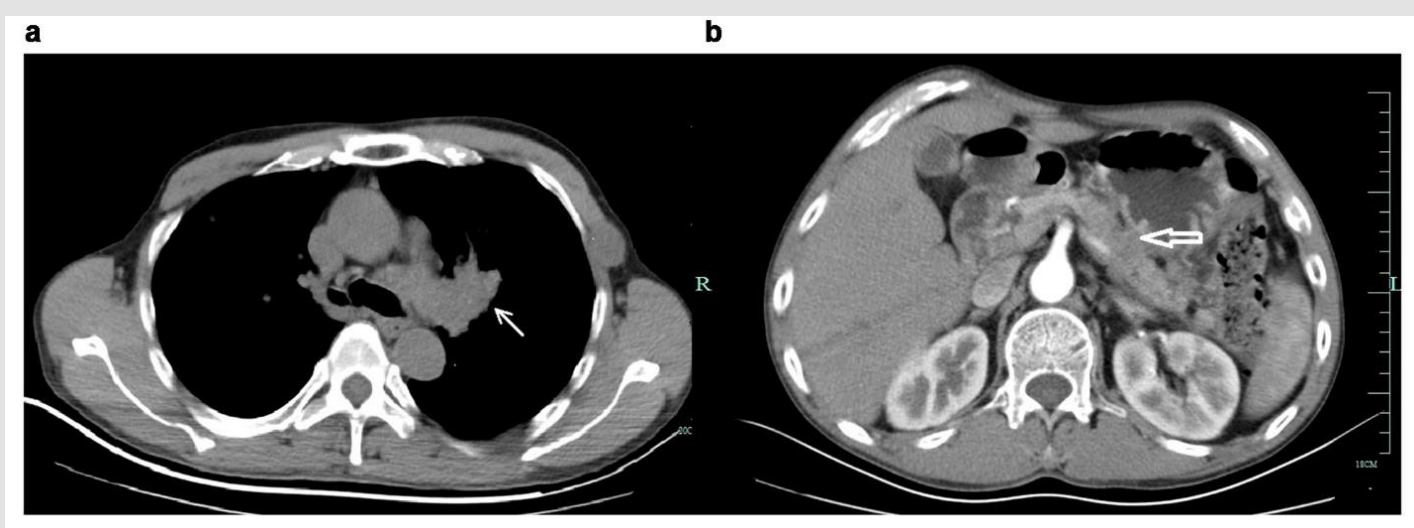

Figure 4: Enhancement CT of the patient after treatment.

a. Mass in the left lung was shrunken after treatment ( $)$.

b. Lesions in the body and tail of enlarged pancreas were shrunken after treatment $(\longleftarrow)$.

\section{Discussion}

Barnard has first reported neuroendocrine tumors in 1926 [4], and occurred in all parts of the body, including gastrointestinal tract, pancreas, bile duct, liver, lung, etc. Gastrointestinal neuroendocrine tumors are the most common neoplasms, accounting for $55-70 \%$ of all neuroendocrine tumors [5]. Recently, the incidence and prevalence of lung neuroendocrine tumors have increased linearly, and previous literature has reported as 1.35 1.57/ 100,000 people [6-7], accounting for $25 \%$ of primary lung tumors and $20 \% \sim 25 \%$ of all neuroendocrine tumors [8]. Lung neuroendocrine tumors were divided into four histological types: small cell lung cancer (SCLC) (20\%), large cell neuroendocrine cancer (LCNEC) (3\%), typical carcinoid (TC) (2\%) and atypical carcinoid (AC) (0.2\%) [9]. SCLC is associated with smoking, mainly occurs in middle-aged and elderly men, and most commonly occurs in the hilum of lung. The 5-year and 10 -year survival rates are $10 \%$ and $5 \%$, respectively [10]. This patient has a previous risk of long-term smoking, and the primary lesion is located in the left hilum of the lung, which was consistent with the previous report [11].

Primary pulmonary neuroendocrine tumors are more prone to distant metastases, and the most common tissues included liver, bone, brain, and adrenal $[1,12,13]$. But pancreatic metastasis is quite rare, which accounted for about $0.7 \%$ [2]. Most of the metastases are still dominated by pulmonary symptoms, which might be related to the fact that the tumor cells metastasize to the pancreas through hematogenous or lymphatic routes, but there was no infiltration of the biliopancreatic duct wall. In this case, abdominal symptoms (abdominal pain) were the first symptoms, which were relatively rare, and may be related to the tumor compression of biliopancreatic duct. In addition, different from primary pancreatic cancer, majority of the patients with metastatic pancreatic cancer had normal serum CA19-9 levels [3]. He [14] analyzed 25 patients with pancreatic metastases and found that the CA19-9 levels were significantly increased in only one case of gallbladder cancer and one case of colon cancer with pancreatic metastases. Lung neuroendocrine neoplasms with elevated serum CA19-9 levels are rarely reported in the literature. According to the images of abdominal CT and elevated tumor markers (CA19-9 and CEA), our patient was misdiagnosed with pancreatic cancer. However, according to CT, and combined with patient's advanced malignant status and good general conditions, the possibilities of uncommon tumors should also be considered, suggesting a multidisciplinary discussion. Thus, wide range of tumor properties should be considered during pathological and immunohistochemical examination. In addition, we also needed to consider the possibility that both the pancreas and lungs are primary lesions.

While the evidence supporting pancreatic metastases is as follows:

1. Miltifocal pancreatic masses

2. Slightly elevated CA19-9

3. Good condition and

4. The imaging finding supported the lung lesion tended to be primary.

For the limitaion of medical equipment (especially the endoscopic ultrasonography) in county hospital, and the the patient was reluctant to be transferred to the superior hospital, it is difficult to obtain a biopsy of lung and pancreatic lesion. The differentiation points of SCLC and other neuroendocrine tumor types included the cell size, the ratio of nucleoli to cytoplasm and the presence of nucleoli. Immunohistochemical markers of pulmonary neuroendocrine tumors were CgA (chromogranin A), Syn (synaptophysin), CD56, thyroid transcription factor 1 (TTF1), ki-67, et al. TTF-1 is a specific molecular marker of lung tissue 
and thyroid gland [15-17]. Syn, CD56 and CgA were considered as neuroendocrine markers. Among these, CD56 is more sensitive, while CgA and Syn are more specific $[18,19]$. The expression rates of Syn, CgA and CD56 were 91\%, 54\% and 87\% in carcinoid (including typical carcinoid and atypical carcinoid), 91\%, 54\% and 87\% in SCLC, and 76\%, 97\% and 92\% in LCNEC, respectively [20]. In this patient, CgA, Syn, CD56, TTF1 and other lung neuroendocrine tumor markers were all positive, while CK7 and other gland sources and exocrine differentiation markers like Napsin-A were negative, and so the pancreatic sources were not considered. The diagnosis of left upper lobe SCLC remained clear.

The staging of SCLC was according to the AJCC TNM method and Veterans Administration Cancer Study Group (VALG) phase II method [21,22]. Surgery (T1-2) and concurrent chemoradiotherapy was performed within a limited stage, and chemoradiotherapy was performed for extensive stage [21-24]. The chemotherapy regimen used was platinum combined with etoposide or irinotecan [2528]. Patients with brain metastases should be treated with wholebrain radiation therapy, while prophylactic brain radiotherapy should be used by considering the disease carefully [29,30]. The brain metastasis of the patient was in extensive stage and received partial remission after 6 courses of etoposide and cisplatin (EP) chemotherapy, followed by whole-brain radiotherapy. After the first-course chemotherapy, the patient's symptoms were improved. Follow-up CT after four courses of chemotherapy showed that the lesions in the lung, pancreas and left chest wall were obviously shrunken. However, the patient's lung lesions developed later and eventually died of respiratory failure. The total survival time is 9 months.

\section{Conclusion}

We herein reported a case of pancreatic metastases from small cell carcinoma of the lung with abdominal symptoms and slightly elevated serum CA19-9 levels. The possibility of other rare tumors should be considered in patients with multiple systemic metastases but were in good general condition. Pathology and immunohistochemistry should adequately used by considering such situations.

\section{Declaration}

\section{Ethics Approval and Consent to Participate}

The study protocol was approved by the Ethics Committees of the people's hospital of Suichang county, and all participants provided written informed consent.

\section{Consent for Publication}

Not applicable.

\section{Availability of Data and Materials}

The datasets used and/or analyzed during the current study are available from the corresponding author on reasonable request.

\section{Competing Interests}

All authors declare that they have no any conflict of interests.

\section{Funding}

The study supported by the Zhejiang Medical and Health Science and Technology Plan Project (No. 2019KY396). The funders had no role in study design, data collection and analysis, decision to publish, or preparation of the manuscript

\section{Author's Contribution}

WH W and YL T collected information and drafted the manuscript. All authors read and approved the final manuscript.

\section{Acknowledgement}

This case report was supported by the department of general medicine of the Second Affiliated Hospital of Zhejiang University, School of Medicine, and the department of internal medicine, radiology and pathology of the People's Hospital of Suichang County. We express our gratitude to all the participants for their support. Written informed consent was obtained form the patient for publication of this report.

\section{References}

1. Kalemkerian GP, Akerley W, Bogner P, Hossein Borghaei, Laura Qm Chow, et al. (2013) Small cell lung cancer. Journal of the National Comprehensive Cancer Network : JNCCN 11(1): 78-98.

2. Duan J, Wan R, Shen J, Xuyi Liu, Jie Wang, et al. (2017) [Clinical Characteristics of Lung Cancer with Pancreatic Metastases]. Zhongguo fei ai za zhi $=$ Chinese journal of lung cancer 20(8): 511-515.

3. Teich N, Kleeff J, Lochs H, Joachim Mössner, Volker Keim, et al. (2005) The presence of the proteolysis-inducing factor in urine does not predict the malignancy of a pancreatic tumour. BMC gastroenterology 5: 20 .

4. Barnard WG (1926) The nature of the "oat-celled sarcoma" of the mediastinum. J Pathol Bacteriol 29(3): 241-244.

5. (2011) [Chinese pathologic consensus for standard diagnosis of gastrointestinal and pancreatic neuroendocrine neoplasm]. Zhonghua Bing Li Xue Za Zhi = Chinese journal of pathology 40: 257-262.

6. Filosso PL, Asamura H, Brunelli A, Mariano Garcia Yuste, Eric Lim (2014) Knowledge of pulmonary neuroendocrine tumors: where are we now? Thoracic surgery clinics 24: ix-xii.

7. Gridelli C, Rossi A, Airoma G, Roberto B, Raffaele C, et al. (2013) Treatment of pulmonary neuroendocrine tumours: state of the art and future developments. Cancer treatment reviews 39(5): 466-472.

8. Yao JC, Hassan M, Phan A, Cecile Dagohoy, Colleen Leary, et al. (2008) One hundred years after "carcinoid": epidemiology of and prognostic factors for neuroendocrine tumors in 35,825 cases in the United States. Journal of clinical oncology : official journal of the American Society of Clinical Oncology 26(18): 3063-3072.

9. Asamura H, Kameya T, Matsuno Y, Masayuki Noguchi, Hirohito Tada, et al. (2006) Neuroendocrine neoplasms of the lung: a prognostic spectrum. Journal of clinical oncology : official journal of the American Society of Clinical Oncology 24(1): 70-76.

10. Teng X, Zhao M, Lai M (2016) [Update of pathological diagnosis of pulmonary neuroendocrine tumor]. Zhejiang da xue xue bao Yi xue ban = Journal of Zhejiang University Medical sciences 45(1): 36-44.

11. Travis WD, Brambilla E, Nicholson AG, Yasushi Yatabe, John H M Austin, et al. (2015) The 2015 World Health Organization Classification of Lung 
Tumors: Impact of Genetic, Clinical and Radiologic Advances Since the 2004 Classification. Journal of thoracic oncology : official publication of the International Association for the Study of Lung Cancer 10(9): 12431260 .

12. Huang Q, Muzitansky A, Mark EJ (2002) Pulmonary neuroendocrine carcinomas. A review of 234 cases and a statistical analysis of 50 cases treated at one institution using a simple clinicopathologic classification. Archives of pathology \& laboratory medicine 126(5): 545-553.

13. Song L, Zhai X, Yu S, Yihui Ma, Feng Wang, et al. (2019) Clinical analysis of 547 patients with neuroendocrine tumors in a Chinese population: A single-center study. Cancer medicine 8(8): 3729-3737.

14. He X, Liu Y, Zhang H, Xiao M (2010) Diagnosis and Treatment of Metastasized Pancreatic Tumor: a Report of 25 Cases. Chinese Journal Of Clinical Oncology, pp. 651-653.

15. Li Z, Dacic S, Pantanowitz L, Khalbuss WE, Nikiforova MN, et al. (2014) Correlation of cytomorphology and molecular findings in EGFR+, KRAS+, and ALK+ lung carcinomas. American journal of clinical pathology 141(3): 420-428.

16. Zhao LH, Chen C, Mao CY, Xiao He, Fu ping, et al. (2019) Value of SATB2, ISL1, and TTF1 to differentiate rectal from other gastrointestinal and lung well-differentiated neuroendocrine tumors. Pathology, research and practice 215:152448.

17. Wang HY, Li ZW, Sun W, Xin Yang, Li-Xin Zhou, et al. (2019) Automated quantification of Ki-67 index associates with pathologic grade of pulmonary neuroendocrine tumors. Chinese medical journal 132(5): 551-561.

18. Korse CM, Taal BG, Vincent A, Marie Louise F van Velthuysen, Paul Baas et al. (2012) Choice of tumour markers in patients with neuroendocrine tumours is dependent on the histological grade. A marker study of Chromogranin A, Neuron specific enolase, Progastrin-releasing peptide and cytokeratin fragments. European journal of cancer (Oxford, England : 1990) 48(5): 662-671.

19. Kashiwagi K, Ishii J, Sakaeda M, Yuu Arimasu, Hiroaki Shimoyamada, et al. (2012) Differences of molecular expression mechanisms among neural cell adhesion molecule 1, synaptophysin, and chromogranin A in lung cancer cells. Pathology international 62(4): 232-245.

20.Zhou DM, Sun Y, Li XH (2013) [Diagnostic value of microtubuleassociated protein-2 in small cell lung carcinoma: an analysis of 240 biopsy cases]. Zhonghua bing li xue za $\mathrm{zhi}=$ Chinese journal of pathology 42(5): 321-324.

ISSN: 2574-1241

DOI: 10.26717/BJSTR.2021.37.006036

Yuling Tong MS. Biomed J Sci \& Tech Res

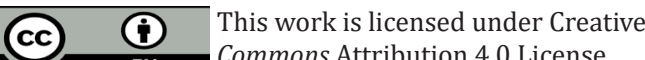

Submission Link: https://biomedres.us/submit-manuscript.php
21. Jett JR, Schild SE, Kesler KA, Kalemkerian GP (2013) Treatment of small cell lung cancer: Diagnosis and management of lung cancer, 3rd ed: American College of Chest Physicians evidence-based clinical practice guidelines. Chest 143: e400S-e419S.

22. Kalemkerian GP, Gadgeel SM (2013) Modern staging of small cell lung cancer. Journal of the National Comprehensive Cancer Network : JNCCN 11(1): 99-104.

23. Warde P, Payne D (1992) Does thoracic irradiation improve survival and local control in limited-stage small-cell carcinoma of the lung? A metaanalysis. Journal of clinical oncology : official journal of the American Society of Clinical Oncology 10(6): 890-895.

24. Pignon JP, Arriagada R, Ihde DC, D H Johnson, M C Perry, et al. (1992) A meta-analysis of thoracic radiotherapy for small-cell lung cancer. The New England journal of medicine 327(23): 1618-1624.

25. Noda K, Nishiwaki Y, Kawahara M, Shunichi Negoro, Takahiko Sugiura, et al. (2002) Irinotecan plus cisplatin compared with etoposide plus cisplatin for extensive small-cell lung cancer. The New England journal of medicine 346(2): 85-91.

26. Schmittel A, Fischer von Weikersthal L, Sebastian M, P Martus, K Schulze, et al. (2006) A randomized phase II trial of irinotecan plus carboplatin versus etoposide plus carboplatin treatment in patients with extended disease small-cell lung cancer. Annals of oncology : official journal of the European Society for Medical Oncology 17(4): 663-667.

27. Spigel DR, Townley PM, Waterhouse DM, Liang Fang, Ibrahim Adiguzel , et al. (2011) Randomized phase II study of bevacizumab in combination with chemotherapy in previously untreated extensive-stage small-cell lung cancer: results from the SALUTE trial. Journal of clinical oncology : official journal of the American Society of Clinical Oncology 29(16): 2215-2222.

28. Schulze AB, Evers G, Kerkhoff A, Michael Mohr ,Christoph Schliemann, et al. (2019) Future Options of Molecular-Targeted Therapy in Small Cell Lung Cancer. Cancers 11(5): 690.

29. Tsao MN, Lloyd N, Wong RK, Wei Xu, Rebecca Ks Wong, et al. (2012) Whole brain radiotherapy for the treatment of newly diagnosed multiple brain metastases. The Cochrane database of systematic reviews: Cd003869.

30. Takahashi T, Yamanaka T, Seto T, Hideyuki Harada, Hiroshi Nokihara, et al. (2017) Prophylactic cranial irradiation versus observation in patients with extensive-disease small-cell lung cancer: a multicentre, randomised, open-label, phase 3 trial. The Lancet Oncology 18(5): 663671.

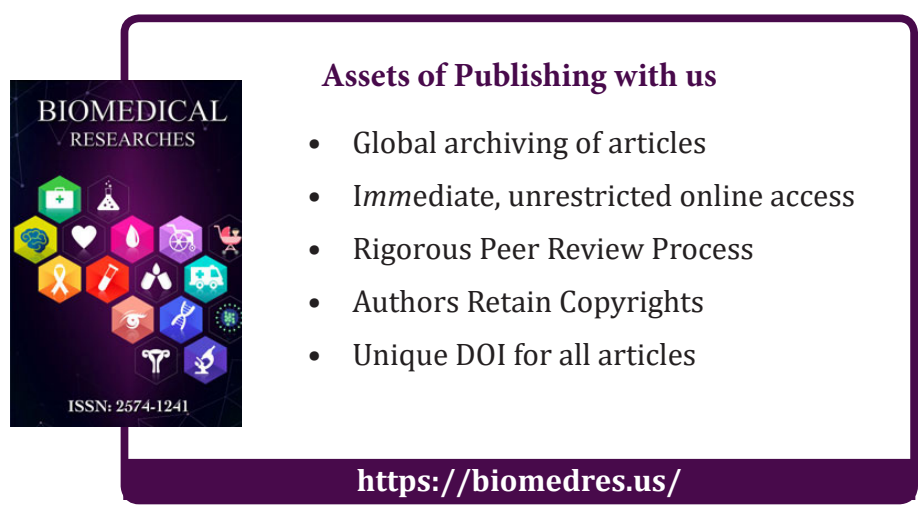

